\title{
Post-treatment with the GLP-1 analogue liraglutide alleviate chronic inflammation and mitochondrial stress induced by Status epilepticus
}

\author{
Rui-Fang Wang ${ }^{1}$, Guo-Fang Xue ${ }^{1}$, Christian Hölscher ${ }^{1,2}$, Miao-Jing Tian ${ }^{1}$, Peng Feng ${ }^{1}$, Ji-Ying \\ Zheng ${ }^{1}$ and Dong-Fang $\mathrm{Li}^{1}$ \\ 1 Department of Neurology, the Second Affiliated Hospital of Shanxi Medical University, No.382 Wuyi \\ Road, Taiyuan 030001, Shanxi Province, China \\ 2 Division of Biomedical and Life Science, Faculty of Health and Medicine, Lancaster University, Lancaster \\ LA14YQ, UK
}

accepted in 'Epilepsy'

Corresponding authors:

Guofang Xue, Department of Neurology, the Second Affiliated Hospital of Shanxi Medical University, No.382 Wuyi Road, Taiyuan 030001, Shanxi Province, China, email: xueguofangty@163.com

Dongfang Li, Department of Neurology, the Second Affiliated Hospital of Shanxi Medical University, No.382 Wuyi Road, Taiyuan 030001, Shanxi Province, China, email: lidongfang898@163.com 


\begin{abstract}
Glucagon-like peptide-1(GLP-1) is a growth factor that has neuroprotective and anti-inflammatory properties. The protease resistant GLP-1 analogue liraglutide has been shown to be neuroprotective in previous studies in animal models of Alzheimer's disease or Parkinson's disease. Status epilepticus (SE) is a complex disorder, involving many underlying pathological processes, including excitotoxic and chronic inflammatory events. The present pilot study aims to investigate whether liraglutide alleviates the chronic inflammation response and mitochondrial stress induced by SE in the lithium-pilocarpine animal model. We found that treatment with $25 \mathrm{nmol} / \mathrm{kg}$. i.p. once-daily after the induction of SE for 7 days reduced chronic inflammation as shown by reduced numbers of activated microglia and astrocytes, and reduced levels of TNF- $\alpha$ and IL-1ß in the hippocampus. The mitochondrial stress marker BAX was reduced and the survival factor Bcl-2 was enhanced by liraglutide. Blood glucose levels were not affected by liraglutide. We show for the first time that liraglutide can reduce the chronic inflammation and mitochondrial stress induced by SE, and the results suggest that GLP-1 receptor agonists such as liraglutide have restorative and protective effects in the brain after SE and could serve as a potential treatment.
\end{abstract}

Key words: liraglutide, GLP-1, apoptosis, inflammation, neuroprotection, insulin 


\section{Introduction}

Status epilepticus (SE) is a neurological condition that leads to substantial morbidity and mortality [1, 2]. On average, about 1/3-1/2 SE patients will get worse, and about 2/5 refractory epilepsy patients will die [3-5]. Surviving patients may suffer from cognitive disorders or neuronal impairments [6-8]. At present, benzodiazepines such as lorazepam and diazepam are widely applied as first-line treatments for SE [9]. Although injections of diazepam $(10 \mathrm{mg} / \mathrm{kg}$ ) after the first seizure can effectively stop seizures and reduce mortality, it fails to impact on the resulting neurodegeneration $[10,11]$. The currently available antiepileptic drugs can only alleviate symptoms of seizure, but not reduce the neurodegenerative processes induced by SE.

Chronic inflammation of the brain also plays a crucial role in epileptogenesis. Inflammation of the hippocampus involves activated glial cells and the release of specific inflammatory cytokines [12, 13]. Activated microglia and astrocytes secrete pro-inflammatory cytokines such as interleukin $1 \beta$ (IL-1 $\beta$ ), tumour necrosis factor- $\alpha$ (TNF- $\alpha$ ) and chemoattractant (MCP1) which can decrease seizure threshold [13-16]. In addition, peripheral immune cells including leukocytes, monocytes/ macrophages and granulocytes infiltrate the CNS after SE which contribute to the chronic epilepsy [17-19]. In the SE brain, astrocytic glutamate release leads to excessive accumulation in extracellular spaces [20-22]. Increased levels of glutamate will increase neuronal activity and enhance excitotoxicity by production of ROS/RNS [23-25]. Therefore, anti-inflammatory agents or antioxidants may be a promising strategy to reduce neuronal damage after SE $[4,15,26]$.

Liraglutide, a Glucagon-like peptide-1 (GLP-1) analogue, has been developed clinically for the treatment of type 2 diabetes mellitus (T2DM). The drug has a good safety profile with minimal adverse reaction [27, 28]. GLP-1 receptors (GLP-1R) are widely expressed in the brain on neurones, cell bodies and dendrites in the brain [29-31]. The GLP-1 analogue liraglutide can cross the blood-brain barrier (BBB) and activate GLP-1Rs $[32,33]$. Liraglutide has shown neuroprotective effects in a range of animal models of neurodegenerative disorders, such as Alzheimer's disease [33-35], Parkinson's disease [36, 37], traumatic brain injury [38, 39], motor neurone disease/ ALS [40], or stroke [41, 42]. Furthermore, liraglutide has anti-inflammatory properties in models of chronic inflammation of the brain [33, 35, 43, 44]. A pilot clinical trial has shown protective effects in patients with Alzheimer's disease [45], and another pilot study has shown improvements in cognitive performance and in MRI brain scans of people with mood disorders [46, 47]. In addition, the GLP-1 receptor agonist exendin-4 has shown good protective effects in a pilot study in Parkinson's patients $[48,49]$. A recently published phase II placebo- controlled double blind trial confirmed the initial results and 
showed that exendin-4 stopped disease progression [50]. This proof of concept demonstrates that the approach of activating GLP-1 receptors as a neuroprotective treatment is valid and translates into the clinic. Therefore, liraglutide or other GLP-1 analogues have the potential to alleviate the pathological processes found in SE. We therefore tested liraglutide in the pilocarpine model of SE [51] at a dose that previously showed neuroprotective effects in the CNS [33]. We show for the first time that liraglutide has clear neuroprotective properties in this animal model.

\section{Materials and methods}

Animals

Male Sprague-Dawley (SD) rats weight 200-250 g were used. All animals were provided by the Beijing Vital River Laboratory Animal Technology. And they were housed under controlled temperature $\left(22 \pm 3 \mathrm{C}^{\circ}\right)$, humidity (50-55\%) and 12-h light/dark cycles with free access to food and water ad libitum. The work was licenced by the ethics committee of Shanxi province. All experimental procedures were conducted in accordance with the National Institutes of Health(NIH) guideline (National Institutes of Health Publications, No. 80-23, revised 1978).

\section{Liraglutide}

Liraglutide (Peptide Purity: 95.77\%) was obtained from Chinapeptides Ltd (Shanghai, China). The purity of the peptide was confirmed by reversed-phase HPLC and characterised using matrix assisted laser desorption/ionisation time of flight (MALDI-TOF) mass spectrometry.

\section{Induction of SE}

The experimental rats were injected with lithium chloride (127 mg/kg, ip., Sigma-Aldrich, USA) 20 hours before atropine sulfate $(1 \mathrm{mg} / \mathrm{kg}$, i.p., Tianjin Jinyao, China) which reduced the peripheral cholinergic effects of pilocarpine. Then, 30 minutes later, pilocarpine hydrochloride (30mg/kg, i.p., MedChem Express, USA) was administered. Each animal's behaviour was evaluated according to Racine's scale [52], and only those rats that exhibited ongoing convulsive seizures (level 4-5) for $30 \mathrm{~min}$ without recovery between seizures would be used as SE model. Diazepam (10 mg/kg, i.p., Tianjin Jinyao, China) was used to treat the animals to terminate the convulsive seizures one hour after onset of SE. Based on the high mortality of pilocarpine-induced SE model, we would add animals to guarantee the numbers of each group. 
The 54 rats were divided into three groups: (I) saline treated control group $(n=6)$; (II) pilocarpine treated group ( $n=24)$; (III) pilocarpine+liraglutide group $(n=24)$. The pilocarpine group and pilocarpine+liraglutide group were divided into four subgroups $(n=6)$ according to the different time points (12h, 1days, 3days, 7days) after termination of SE. Following the termination, liraglutide $(25 \mathrm{nmol} / \mathrm{kg}$, ip.) was administered immediately once -daily in the pilocarpine+liraglutide group, while the pilocarpine group rats were treated with $0.9 \% \mathrm{NaCl}$ saline instead. The dose of liraglutide was based on the studies showing beneficial effects in AD or PD $[35,53,54]$.

\section{Glucose measurements}

Blood samples from the tail vein were used for the glucose test. Blood glucose levels were tested before sacrifice at different time points (12 h, 1d, 3d, 7d after termination of SE) by a Sannuo blood glucose meter (Sinocare Inc. China). Repeated measurements were taken three times to reduce error and the average was taken as the blood glucose level.

Western blot

Three rats in each subgroup were sacrificed. Rats were anesthetized with $5 \%$ chloral hydrate ( $5 \mathrm{ml} / \mathrm{kg}$, ip.,). After cardiac perfusion with cold $0.9 \% \mathrm{NaCl}$, the brain was dissected on ice and hippocampi were removed. Hippocampus tissue was homogenised in ice cold RIPA buffer (Beyotime Institute of Biotechnology, China) added phenyl-methylsulfonyl fluoride (PMSF) and centrifuged $12000 \mathrm{rpm} / \mathrm{min}(=3200 \mathrm{~g})$ for $10 \mathrm{~min}$ at $4 \mathrm{C}^{\circ}$. Then, the supernatant was taken for detection of protein concentration by BCA Protein Assay Kit (Boster Institute of Biotechnology, China). After supernatant was mixed with loading buffer and boiled for $10 \mathrm{~min}$, every sample that contained 20 ug protein was separated by $12 \%$ sodium dodecyl sulphate polyacrylamide gel electrophoresis (SDA-PAGE) and then transferred to polyvinylidene difluoride (PVDF) membranes. Next, the membranes were blocked with $5 \%$ non-fat milk in $4 \mathrm{C}^{\circ}$ for one night to prevent nonspecific binding. Primary antibodies incubated overnight at $4 \mathrm{C}^{\circ}$ with rabbit anti-rat $\mathrm{Bcl}-2(1: 1000$, bioworld technology, USA), rabbit anti-rat $\operatorname{Bax}(1: 1000$, Bioworld technology, USA), rabbit anti-rat IL-1 $\beta \quad(1: 1000$, bioworld technology, USA), rabbit anti-rat TNF- $\alpha$ (1:1000, Abcam, UK) and rabbit anti-rat $\beta$-actin (1:3000, Bioworld, USA) diluted in PBS. After washing three times (10 min per wash) in TBST, membranes were incubated in goat anti-rabbit $\lg G$ with horseradish peroxidise conjugate (1:3000, Boster Institute of Biotechnology, China) for 2 hours and then washed three times in TBST. The protein bands were detected by ECL-enhanced chemiluminescence (Boster Institute of Biotechnology, China) and analysed by Image Lab 3.0 System.

Immunohistochemistry

After anesthetized with $5 \%$ chloral hydrate $(5 \mathrm{ml} / \mathrm{kg}$, ip., $)$, the rats $(n=3)$ were perfused with ice-cold $0.9 \%$ 
saline followed by 4\% paraformaldehyde (PFA). Then brains were obtained and postfixed in 4\% PFA for 24 hours at $4 \mathrm{C}^{\circ}$. After treatment with alcohol and xylene by a Leica TP1020 histology system, brains were embedded in paraffin and $5 \mu \mathrm{m}$ sections were cut on a Leica (Germany) microtome. The brain sections were incubated with $3 \%$ hydrogen peroxide $\left(\mathrm{H}_{2} \mathrm{O}_{2}\right)$ for $10 \mathrm{~min}$ at room temperature $(\mathrm{RT})$. Then, sections were treated with primary antibodies including rabbit anti-rat Bcl-2 (1:50, Bioworld technology, USA), rabbit anti-rat Bax (1:50, bioworld technology, USA), rabbit anti-rat IBA1 (1:100, Abcam, UK), rabbit anti-rat GFAP (1:100, Abcam, UK) and rabbit anti-rat TNF- $\alpha$ (1:1000, Abcam, UK) overnight at $4 C^{\circ}$, then with goat anti-rabbit $\lg$ (1:500, ZSGB-BIO) and incubated at $37 C^{\circ}$ for 1 hour. The immunoreactivity was visualized with DAB (Boster Institute of Biotechnology, China) colour reaction. The CA1 region of the hippocampus was visualized with an Olympus BX51 (Olympus, Japan) microscope. For the analysis, stereological rules were applied. Three sections per brain were used for histology and two images were chosen randomly from each section (right and left hippocampus) to count the number of positive cells. The values obtained by the six analyzed images from one brain tissue were averaged before to enter the statistical analysis. The surface area analysed per disector was $1.10 \mathrm{~mm}^{2}$. Image-Pro Plus 6.0 (Media Cybernetics, Silver Spring, USA) was applied for automated cell count. Thresholds for object size and intensity of the stain used were set to count the cells following the instructions of the manufacturer. The mean value of the positive cell numbers from images of each rat was used for statistical analysis.

Statistical analysis

All data were expressed as mean \pm standard error (SEM). Statistical analyse software PRISM 5.0 (GraphPad software, USA) was used for analysis. Data were analysed by one-way or two-way analysis of variance (ANOVA), followed by Tukey multiple comparison post hoc tests. $P<0.05$ was defined as statistically significant.

\section{Results}

Measurements of plasma glucose levels

A repeated measure two-way ANOVA did not find a difference between blood glucose levels in the two groups. Neither time $(P>0.05, F=1.24)$ nor drug treatment $(P>0.05, F=1.35)$ was significantly different (see Fig. 1).

Histological analysis: 


\section{Activation of microglia and astrocytes in the hippocampus}

When analysing activated microglia numbers (IBA-1 positive cells) in the hippocampus, a two-way ANOVA found a significant difference between drug treatment $(P<0.0001, D F=3, F=280.3)$ and time $(P<0.0001$, $\mathrm{DF}=1, \mathrm{~F}=381)$. Interaction was significant $(\mathrm{P}<0.0001, \mathrm{DF}=3, \mathrm{~F}=216)$. Post-hoc repeated measures Tukey post-hoc tests found differences between groups (see Fig. 2). When measuring activated astrocyte numbers (GFAP positive cells) in the hippocampus, a two-way ANOVA found a significant difference between drug treatment $(P<0.0001, D F=3, F=39)$ and time $(P<0.0001, D F=1, F=19.6)$. Interaction was significant $(P<0.05$, $\mathrm{DF}=3, \mathrm{~F}=4.0$ ). Post-hoc repeated measures Tukey post-hoc tests found differences between groups (see Fig. 3). Treatment with Liraglutide was able to reduce the chronic inflammation response.

\section{Levels of mitochondrial signalling peptides Bcl-2 and BAX in the hippocampus}

When evaluating the levels of $\mathrm{Bcl}-2$ positive cells, a two-way ANOVA found a significant difference between drug treatment $(P<0.0001, D F=3, F=21.3)$ and time $(P<0.005, D F=1, F=9.15)$. Interaction was not significant. Post-hoc repeated measures Tukey post-hoc tests found differences between groups (see Fig. 4).

Liraglutide treatment enhanced the levels of the protective signalling peptide $\mathrm{Bcl} 2$. When evaluating the levels of BAX positive cells, a two-way ANOVA found a significant difference between drug treatment $(P<0.0001, D F=3, F=18.3)$ and time $(P<0.0001, D F=1, F=149.2)$. Interaction was significant $(P<0.0001$, $\mathrm{DF}=3, \mathrm{~F}=9.3$ ). Post-hoc repeated measures Tukey post-hoc tests found differences between groups (see Fig. 5). Liraglutide treatment reduced the levels of the autophagy and apoptosis signalling peptide BAX in the hippocampus.

Western blot analysis:

Liraglutide reduces the levels of pro-inflammatory cytokines

When analysing the levels of TNF- $\alpha$ in the brain, a one-way ANOVA found a significant difference between groups $(P<0.0001, F=19.56$, DF=80). Post-hoc repeated measures Tukey post-hoc tests found differences between groups (see Fig. 6a). When analysing the levels of IL-1ß in the brain, a one-way ANOVA found a significant difference between groups $(P<0.605, F=4.24, D F=80)$. Post-hoc repeated measures Tukey post-hoc tests found differences between groups (see Fig. 6b).

\section{Liraglutide reduces mitochondrial stress}

When analysing the levels of $\mathrm{Bcl}-2$ (B-cell lymphoma 2 peptide) in the brain, a one-way ANOVA found a significant difference between groups $(P<0.0001, F=14.64$, DF=80). Post-hoc repeated measures Tukey post-hoc tests found differences between groups (see Fig. 6c). When analysing the levels of BAX in the 
brain, a one-way ANOVA found a significant difference between groups $(P<0.0001, F=13.7, D F=80)$. Post-hoc repeated measures Tukey post-hoc tests found differences between groups (see Fig. 6d).

\section{Discussion}

Inducing SE in the rats by lithium chloride and pilocarpine treatment induced chronic inflammation in the brain as observed in the increased activation of microglia and astrocytes and the release of the pro-inflammatory cytokines TNF- $\alpha$ and IL-1ß. Furthermore, mitochondrial function is compromised as indicated by the reduced levels of $\mathrm{Bcl}-2$ and the increased levels of $\mathrm{BAX}$. Bcl-2 is a mitochondrial signal that is released when the membrane potential in the mitochondrial cristae is within physiological levels. Bcl-2 blocks autophagy and apoptosis. BAX is released in the mitochondria when the voltage potential in mitochondria is reduced, a sign of dysfunction and damage. BAX can induce the elimination and autophagy of mitochondria, and can furthermore induce apoptosis $[55,56]$. We and others have shown that activation of the GLP-1 receptor can reverse the mitochondrial stress signalling induced by toxins and increase Bcl-2 levels and decrease BAX levels [57-60]. In our study using pilocarpine to induce SE, liraglutide was able to partially reverse the changes in $\mathrm{Bcl}-2$ and BAX levels, indicating that mitochondrial activity was rescued. The chronic inflammation observed in this SE rat model was also much reduced by liraglutide treatment. The number of activated microglia and astrocytes was reduced, and the levels of TNF- $\alpha$ and IL-1ß in the brain were reduced.

Previous studies found that microglia and astrocytes play a key role in the pathophysiology of epilepsy [61]. Activated microglia and astrocytes go through changes in morphology and functions. For example, uncontrolled activation of astrocytes and microglia is related to (BBB disruption and the release of pro-inflammatory cytokines as shown here. The astrocyte cell processes are associated with BBB function by releasing chemical signals to control BBB uptake [62]. Pro-inflammatory cytokines and glutamate released by astrocytes may affect BBB permeability in multiple ways. The dysfunction of BBB- induced brain extravasation of serum albumin can lead to glial activation, inflammation and synaptogenesis which increases excitability and promotes epileptogenesis [63-65]. In addition, interleukin-1ß released by astrocytes can damage BBB integrity during seizures [66]. The inflammation response to acute seizures leads to a rapid increase in inflammatory cytokine levels including IL-1 $\beta$, TNF- $\alpha$, IL- 6 and prostaglandins released by microglia and astrocytes $[13,14,26,67]$. Following the seizures, activated glial cells release high amounts of cytokines in the hippocampus which can be detected at 12 hours, 1 days, 3 days, 7 days later. Our experimental results also observed that the activation of microglia and astrocytes in the CA1 region of hippocampus is persistent. This finding confirms previous studies $[26,68]$. The release of 
inflammatory cytokines plays a vital role in the development of epileptogenesis $[13,16]$. IL-1 $\beta$ can increase the NMDA-mediated inward $\mathrm{Ca}^{2+}$ currents, which induce the release of glutamate and modulates synaptic activity $[62,69]$. Increased TNF- $\alpha$ induces a rapid upregulation of AMPA receptor levels that lack the GluR2 subunit which also enhances the membrane permeability of $\mathrm{Ca}^{2+}$. These changes promote neuronal excitability. In addition, the IL-1 $\beta$ and TNF- $\alpha$ released by glia increase BBB permeability. The inhibition of IL-1 $\beta$ or TNF- $\alpha$ has been shown to prevent epileptic activity $[70,71]$. Activation of the IL-1 receptor/Toll-like receptor can decrease the threshold of seizures $[13,72,73]$. In addition, activated astrocytes also regulate extracellular potassium levels and neurotransmitter levels [13]. Therefore, the prevention or reduction of astrocyte activation is part of the therapeutic effect of liraglutide. Activated microglia can promote astrocytic activation, and activated astrocytes feed back to microglia by releasing pro-inflammatory cytokines [62]. Liraglutide decreased the expression of IL-1 $\beta$ and TNF- $\alpha$ in the hippocampus, which contributes to the neuroprotective effect of this drug. Our results confirm previous studies that demonstrated protective effects of liraglutide in the PTZ kindling -induced epilepsy model and the corneal kindling epilepsy model.

Pre-treatment with liraglutide prevented the seizure severity, normalised behavioural activity, reduced oxidative stress, and normalised the levels of key neurotransmitters in the brain $[74,75]$. In addition, liraglutide has shown neuroprotective effects in a range of preclinical studies of other neurodegenerative disorders [76], such as Alzheimer's disease [33-35], Parkinson's disease [36, 37], traumatic brain injury [38, 39], motor neurone disease / ALS [40], or stroke [41, 42]. A pilot clinical trial has shown protective effects of liraglutide in patients with Alzheimer's disease [45], and another pilot study showed clear improvements in cognitive function and in MRI brain scans of key brain areas [46, 47]. Importantly, exendin-4, a different GLP-1R agonist with good neuroprotective effects has been tested in Parkinson's patients with good results $[48,49]$. A phase II double-blind, placebo controlled trial that recently finished confirmed the results from the pilot study, demonstrating that the strategy of activating GLP-1 receptors in the brain is a viable strategy in treating neurodegenerative disorders [50]. Consequently, liraglutide is currently in a phase II test in Alzheimer's patients (clinical trial identifier NCT01843075) and in a phase II trial in Parkinson's patients (NCT02953665).

In conclusion, our results demonstrate that liraglutide alleviates brain inflammation and mitigates mitochondrial apoptotic signalling in a pilocarpine induced SE rat model. This suggests that liraglutide may be effective in treating patients that have experienced SE.

\section{Acknowledgements}

The work has been supported by China Association Against Epilepsy (Grant NO.2017007), National Natural Science Foundation of China (Grant NO.81601038), Shanxi Science and Technology Department (Grant 
NO.2014021038-4) and Shanxi Medical University Innovation and Entrepreneurship Foundation of China (Grant NO.057602). The authors declare no conflict of interest.

\section{References}

1. Fountain NB. Status epilepticus: Risk factors and complications. Epilepsia 2000; 41: S23-S30.

2. Neligan A, Shorvon SD. Prognostic factors, morbidity and mortality in tonic-clonic status epilepticus: a review. Epilepsy Res 2011; 93: 1-10.

3. Chen WB, Gao R, Su YY, Zhao JW, Zhang YZ, Wang L, et al. Valproate versus diazepam for generalized convulsive status epilepticus: a pilot study. Eur J Neurol 2011; 18: 1391-6.

4. Holtkamp M. Predictors and prognosis of refractory status epilepticus treated in a neurological intensive care unit. Journal of Neurology, Neurosurgery \& Psychiatry 2005; 76: 534-9.

5. Vooturi S, Jayalakshmi S, Sahu S, Mohandas S. Prognosis and predictors of outcome of refractory generalized convulsive status epilepticus in adults treated in neurointensive care unit. Clinical Neurology and Neurosurgery 2014; 126: 7-10.

6. Kantanen AM, Reinikainen M, Parviainen I, Kalviainen R. Long-term outcome of refractory status epilepticus in adults: A retrospective population-based study. Epilepsy Res 2017; 133: 13-21.

7. Hesdorffer DC, Logroscino G, Cascino G, Annegers JF, Hauser WA. Risk of unprovoked seizure after acute symptomatic seizure: effect of status epilepticus. Ann Neurol 1998; 44: 908-12.

8. Ambrogini P, Minelli A, Galati C, Betti M, Lattanzi D, Ciffolilli S, et al. Post-Seizure $\alpha$-Tocopherol Treatment Decreases Neuroinflammation and Neuronal Degeneration Induced by Status Epilepticus in Rat Hippocampus. Molecular Neurobiology 2014; 50: 246-56.

9. Trandafir CC, Pouliot WA, Dudek FE, Ekstrand JJ. Co-administration of subtherapeutic diazepam enhances neuroprotective effect of COX-2 inhibitor, NS-398, after lithium pilocarpine-induced status epilepticus. Neuroscience 2015; 284: 601-10.

10. Pouliot W, Bialer M, Hen N, Shekh-Ahmad T, Kaufmann D, Yagen B, et al. A comparative electrographic analysis of the effect of sec-butyl-propylacetamide on pharmacoresistant status epilepticus. Neuroscience 2013; 231: 145-56.

11. Pitkanen A, Kharatishvili I, Narkilahti S, Lukasiuk K, Nissinen J. Administration of diazepam during status epilepticus reduces development and severity of epilepsy in rat. Epilepsy Res 2005; 63: 27-42. 12. Ravizza T, Balosso S, Vezzani A. Inflammation and prevention of epileptogenesis. Neuroscience Letters 2011; 497: 223-30.

13. Vezzani A, Aronica E, Mazarati A, Pittman QJ. Epilepsy and brain inflammation. Exp Neuro/ 2013; 244: 11-21. 
14. Vezzani A, Ravizza T, Balosso S, Aronica E. Glia as a source of cytokines: implications for neuronal excitability and survival. Epilepsia 2008; 49 Suppl 2: 24-32.

15. Puttachary S, Sharma S, Stark S, Thippeswamy T. Seizure-Induced Oxidative Stress in Temporal Lobe Epilepsy. BioMed Research International 2015; 2015: 1-20.

16. Vezzani A, French J, Bartfai T, Baram TZ. The role of inflammation in epilepsy. Nat Rev Neurol 2011; 7: 31-40.

17. Fabene PF, Navarro Mora G, Martinello M, Rossi B, Merigo F, Ottoboni L, et al. A role for leukocyte-endothelial adhesion mechanisms in epilepsy. Nature medicine 2008; 14: 1377-83.

18. Varvel NH, Neher J], Bosch A, Wang W, Ransohoff RM, Miller RJ, et al. Infiltrating monocytes promote brain inflammation and exacerbate neuronal damage after status epilepticus. Proceedings of the National Academy of Sciences of the United States of America 2016; 113: E5665-74.

19. Vinet J, Vainchtein ID, Spano C, Giordano C, Bordini D, Curia G, et al. Microglia are less pro-inflammatory than myeloid infiltrates in the hippocampus of mice exposed to status epilepticus. Glia 2016; 64: 1350-62.

20. Fitch MT, Silver J. CNS injury, glial scars, and inflammation: Inhibitory extracellular matrices and regeneration failure. Exp Neurol 2008; 209: 294-301.

21. Seifert G, Carmignoto G, Steinhauser C. Astrocyte dysfunction in epilepsy. Brain Res Rev 2010; 63: 212-21.

22. Sofroniew MV, Vinters HV. Astrocytes: biology and pathology. Acta Neuropathologica 2010; 119: 7-35.

23. Ravizza T, Boer K, Redeker S, Spliet WGM, van Rijen PC, Troost D, et al. The IL-1 $1 \beta$ system in epilepsy-associated malformations of cortical development. Neurobiology of Disease 2006; 24 : 128-43.

24. Ravizza T, Lucas SM, Balosso S, Bernardino L, Ku G, Noe F, et al. Inactivation of caspase-1 in rodent brain: a novel anticonvulsive strategy. Epilepsia 2006; 47: 1160-8.

25. Vezzani A, Moneta D, Conti M, Richichi C, Ravizza T, De Luigi A, et al. Powerful anticonvulsant action of IL-1 receptor antagonist on intracerebral injection and astrocytic overexpression in mice. Proceedings of the National Academy of Sciences 2000; 97: 11534-9.

26. Wang N, Mi X, Gao B, Gu J, Wang W, Zhang Y, et al. Minocycline inhibits brain inflammation and attenuates spontaneous recurrent seizures following pilocarpine-induced status epilepticus. Neuroscience 2015; 287: 144-56.

27. Vilsboll T. Liraglutide: a new treatment for type 2 diabetes. Drugs of today 2009; 45: 101-13.

28. Madsbad S, Schmitz O, Ranstam J, Jakobsen G, Matthews DR. Improved glycemic control with no weight increase in patients with type 2 diabetes after once-daily treatment with the long-acting 
glucagon-like peptide 1 analog liraglutide (NN2211): a 12-week, double-blind, randomized, controlled trial. Diabetes care 2004; 27: 1335-42.

29. Hamilton A, Hölscher C. Receptors for the incretin glucagon-like peptide-1 are expressed on neurons in the central nervous system. NeuroReport 2009; 20: 1161-6.

30. Merchenthaler I, Lane M, Shughrue P. Distribution of pre-pro-glucagon and glucagon-like peptide-1 receptor messenger RNAs in the rat central nervous system. The Journal of comparative neurology 1999; 403: 261-80.

31. Cork SC, Richards JE, Holt MK, Gribble FM, Reimann F, Trapp S. Distribution and characterisation of Glucagon-like peptide-1 receptor expressing cells in the mouse brain. Mol Metab 2015; 4: 718-31. 32. Hunter K, Holscher C. Drugs developed to treat diabetes, liraglutide and lixisenatide, cross the blood brain barrier and enhance neurogenesis. BMC neuroscience 2012; 13: 33-8.

33. McClean P, Parthsarathy V, Faivre E, Hölscher C. The diabetes drug Liraglutide prevents degenerative processes in a mouse model of Alzheimer's disease. The Journal of neuroscience : the official journal of the Society for Neuroscience 2011; 31: 6587-94.

34. Hansen HH, Barkholt P, Fabricius K, Jelsing J, Terwel D, Pyke C, et al. The GLP-1 receptor agonist liraglutide reduces pathology-specific tau phosphorylation and improves motor function in a transgenic hTauP301L mouse model of tauopathy. Brain research 2015; 1634: 158-70.

35. McClean PL, Holscher C. Liraglutide can reverse memory impairment, synaptic loss and reduce plaque load in aged APP/PS1 mice, a model of Alzheimer's disease. Neuropharmacology 2014; 76 Pt A: $57-67$.

36. Badawi GA, Abd El Fattah MA, Zaki HF, El Sayed MI. Sitagliptin and liraglutide reversed nigrostriatal degeneration of rodent brain in rotenone-induced Parkinson's disease. Inflammopharmacology 2017; 25: 369-82.

37. Liu W, Jalewa J, Sharma M, Li G, Li L, Hölscher C. Neuroprotective effects of lixisenatide and liraglutide in the MPTP mouse model of Parkinson' s disease. Neuroscience 2015; 303: 42-50. 38. Li Y, Bader M, Tamargo I, Rubovitch V, Tweedie D, Pick CG, et al. Liraglutide is neurotrophic and neuroprotective in neuronal cultures and mitigates mild traumatic brain injury in mice. Journal of neurochemistry 2015; 135: 1203-17.

39. Hakon J, Ruscher K, Romner B, Tomasevic G. Preservation of the Blood Brain Barrier and Cortical Neuronal Tissue by Liraglutide, a Long Acting Glucagon-Like-1 Analogue, after Experimental Traumatic Brain Injury. PloS one 2015; DOI: 10.1371/journal.pone.0120074.

40. Li Y, Chigurupati S, Holloway HW, Mughal M, Tweedie D, Bruestle DA, et al. Exendin-4 ameliorates motor neuron degeneration in cellular and animal models of amyotrophic lateral sclerosis. PloS one 2012; 7: e32008. 
41. Briyal S, Shah S, Gulati A. Neuroprotective and anti-apoptotic effects of liraglutide in the rat brain following focal cerebral ischemia. Neuroscience 2014; 281C: 269-81.

42. Li PC, Liu LF, Jou MJ, Wang HK. The GLP-1 receptor agonists exendin-4 and liraglutide alleviate oxidative stress and cognitive and micturition deficits induced by middle cerebral artery occlusion in diabetic mice. BMC neuroscience 2016; 17: 37.

43. Parthsarathy $\vee$, Holscher $C$. The type 2 diabetes drug liraglutide reduces chronic inflammation induced by irradiation in the mouse brain. European journal of pharmacology 2013; 700: 42-50. 44. Barreto-Vianna ARC, Aguila MB, Mandarim-de-Lacerda CA. Beneficial effects of liraglutide (GLP1 analog) in the hippocampal inflammation. Metab Brain Dis 2017; DOI: 10.1007/s11011-017-0059-4. 45. Gejl M, Gjedde A, Egefjord L, Møller A, Hansen SB, Vang K, et al. In Alzheimer' s Disease, Six-Month Treatment with GLP-1 Analogue Prevents Decline of Brain Glucose Metabolism: Randomized, Placebo-Controlled, Double-Blind Clinical Trial. Frontiers in aging neuroscience 2016; 8: $1-10$.

46. Mansur RB, Ahmed J, Cha DS, Woldeyohannes HO, Subramaniapillai M, Lovshin J, et al. Liraglutide promotes improvements in objective measures of cognitive dysfunction in individuals with mood disorders: A pilot, open-label study. J Affect Disord 2017; 207: 114-20.

47. Mansur RB, Zugman A, Ahmed J, Cha DS, Subramaniapillai M, Lee Y, et al. Treatment with a GLP-1R agonist over four weeks promotes weight loss-moderated changes in frontal-striatal brain structures in individuals with mood disorders. European neuropsychopharmacology : the journal of the European College of Neuropsychopharmacology 2017; 27: 1153-62.

48. Aviles-Olmos I, Dickson J, Kefalopoulou Z, Djamshidian A, Ell P, Soderlund T, et al. Exenatide and the treatment of patients with Parkinson's disease. The Journal of clinical investigation 2013; 123 : 2730-6.

49. Aviles-Olmos I, Dickson J, Kefalopoulou Z, Djamshidian A, Kahan J, Fmedsci PE, et al. Motor and Cognitive Advantages Persist 12 Months After Exenatide Exposure in Parkinson's Disease. Journal of Parkinson's disease 2014; 4: 337-44.

50. Athauda D, Maclagan K, Skene SS, Bajwa-Joseph M, Letchford D, Chowdhury K, et al. Exenatide once weekly versus placebo in Parkinson's disease: a randomised, double-blind, placebo-controlled trial. The Lancet 2017; 6736: 31585-4.

51. Curia G, Longo D, Biagini G, Jones RS, Avoli M. The pilocarpine model of temporal lobe epilepsy. Journal of neuroscience methods 2008; 172: 143-57.

52. Racine RJ. Modification of seizure activity by electrical stimulation: I. after-discharge threshold. Electroencephalography and Clinical Neurophysiology 1972; 32: 269-79.

53. Yuan Z, Li D, Feng P, Xue G, Ji C, Li G, et al. A novel GLP-1/GIP dual agonist is more effective than 
liraglutide in reducing inflammation and enhancing GDNF release in the MPTP mouse model of Parkinson's disease. Eur J Pharmacol 2017.

54. McClean PL, Jalewa J, Holscher C. Prophylactic liraglutide treatment prevents amyloid plaque deposition, chronic inflammation and memory impairment in APP/PS1 mice. Behavioural brain research 2015; 293: 96-106.

55. Nagatsu T, Mogi M, Ichinose H, Togari A. Cytokines in Parkinson's disease. Journal of neural transmission Supplementum 2000; 58: 143-51.

56. Harada H, Grant S. Apoptosis regulators. Rev Clin Exp Hematol 2003; 7: 117-38.

57. Sharma M, Jalewa J, Holscher C. Neuroprotective and anti-apoptotic effects of Liraglutide on SH-SY5Y cells exposed to Methylglyoxal stress. Journal of neurochemistry 2013; 128: 459-71.

58. Zhang H, Liu Y, Guan S, Qu D, Wang L, Wang X, et al. An Orally Active Allosteric GLP-1 Receptor Agonist Is Neuroprotective in Cellular and Rodent Models of Stroke. PloS one 2016; 11: e0148827. 59. Velmurugan K, Balamurugan AN, Loganathan G, Ahmad A, Hering BJ, Pugazhenthi S. Antiapoptotic actions of exendin-4 against hypoxia and cytokines are augmented by CREB. Endocrinology 2012; 153: 1116-28.

60. Ji C, Xue GF, Lijun C, Feng P, Li D, Li L, et al. A novel dual GLP-1 and GIP receptor agonist is neuroprotective in the MPTP mouse model of Parkinson's disease by increasing expression of BNDF. Brain research 2016; 1634: 1-11.

61. Curia G, Lucchi C, Vinet J, Gualtieri F, Marinelli C, Torsello A, et al. Pathophysiogenesis of mesial temporal lobe epilepsy: is prevention of damage antiepileptogenic? Current medicinal chemistry 2014; 21: $663-88$.

62. Devinsky O, Vezzani A, Najjar S, De Lanerolle NC, Rogawski MA. Glia and epilepsy: excitability and inflammation. Trends Neurosci 2013; 36: 174-84.

63. Heinemann U, Kaufer D, Friedman A. Blood-brain barrier dysfunction, TGFbeta signaling, and astrocyte dysfunction in epilepsy. Glia 2012; 60: 1251-7.

64. Frigerio F, Frasca A, Weissberg I, Parrella S, Friedman A, Vezzani A, et al. Long-lasting pro-ictogenic effects induced in vivo by rat brain exposure to serum albumin in the absence of concomitant pathology. Epilepsia 2012; 53: 1887-97.

65. Gorter JA, van Vliet EA, Aronica E. Status epilepticus, blood-brain barrier disruption, inflammation, and epileptogenesis. Epilepsy \& Behavior 2015; 49: 13-6.

66. Kim JV, Kang SS, Dustin ML, McGavern DB. Myelomonocytic cell recruitment causes fatal CNS vascular injury during acute viral meningitis. Nature 2009; 457: 191-5.

67. Aronica E, Crino PB. Inflammation in epilepsy: clinical observations. Epilepsia 2011; 52: 26-32.

68. Yang F, Liu ZR, Chen J, Zhang SJ, Quan QY, Huang YG, et al. Roles of astrocytes and microglia in 
seizure-induced aberrant neurogenesis in the hippocampus of adult rats. J Neurosci Res 2010; 88 : 519-29.

69. Diemmeler S, Ankarcrona M, Nicotera P, Brüne B. Exogenous Nitric oxide (NO) generation or IL-1b-induced intracellular NO production stimulates inhibitory auto-ADP-ribosylation of glyceraldehyde-3-phosphate dehydrogenase in RINm5F cells. J Immunol 1993; 150: 2964-71.

70. Auvin S, Shin D, Mazarati A, Sankar R. Inflammation induced by LPS enhances epileptogenesis in immature rat and may be partially reversed by IL1RA. Epilepsia 2010; 51 Suppl 3: 34-8.

71. Rao RS, Medhi B, Saikia UN, Arora SK, Toor JS, Khanduja KL, et al. Experimentally induced various inflammatory models and seizure: Understanding the role of cytokine in rat. European Neuropsychopharmacology 2008; 18: 760-7.

72. Maroso M, Balosso S, Ravizza T, Liu J, Aronica E, lyer AM, et al. Toll-like receptor 4 and high-mobility group box-1 are involved in ictogenesis and can be targeted to reduce seizures. Nat Med 2010; 16: 413-9.

73. Zurolo E, lyer A, Maroso M, Carbonell C, Anink J], Ravizza T, et al. Activation of Toll-like receptor, RAGE and HMGB1 signalling in malformations of cortical development. Brain 2011; 134: 1015-32.

74. Koshal P, Kumar P. Neurochemical modulation involved in the beneficial effect of liraglutide, GLP-1 agonist on PTZ kindling epilepsy-induced comorbidities in mice. Molecular and cellular biochemistry 2016; 415: 77-87.

75. Koshal P, Kumar P. Effect of Liraglutide on Corneal Kindling Epilepsy Induced Depression and Cognitive Impairment in Mice. Neurochemical research 2016; 41: 1741-50.

76. Hölscher C. GLP-1 and GIP analogues as novel treatments for Alzheimer' s and Parkinson' $s$ disease. Cardiovasc Endocrinol 2016; 5: 93-8.

\section{Figure captions}

Fig.1: Blood plasma glucose levels of the two groups over time. A two-way repeated measures ANOVA did not find a difference between groups or over time. $\mathrm{N}=6$ per group.

Fig. 2: Number of activated microglia in area CA1 of the hippocampus. A two-way ANOVA found an overall difference between drug treatment and time, and a Tukey repeated measures post-hoc test found differences between time points and drug treatments. ${ }^{* * *}=p<0.001 ;{ }^{* *}=p<0.01 ;{ }^{*}=p<0.05$. Shown are 
representative micrographs. Scale bar $=40 \mu \mathrm{m} . \mathrm{A}=$ Pilo $12 \mathrm{~h} ; \mathrm{B}=$ Pilo day 1 ; $=$ Pilo day $3 ; \mathrm{D}=$ Pilo day7; E=Pilo+Lira 12h; F=Pilo+Lira day1; G=Pilo+Lira day3; H=Pilo+Lira day7; I=untreated. $\mathrm{N}=6$ sections per group.

Fig. 3: Number of activated astrocytes in area CA1 of the hippocampus. A two-way ANOVA found an overall difference between drug treatment and time, and a Tukey repeated measures post-hoc test found differences between time points and drug treatments. ${ }^{* * *}=p<0.001 ;{ }^{* *}=p<0.01 ;{ }^{*}=p<0.05$. Shown are representative micrographs. Scale bar $=40 \mu \mathrm{m} . \mathrm{A}=$ Pilo $12 \mathrm{~h} ; \mathrm{B}=$ Pilo day $1 ; \mathrm{C}=$ Pilo day 3; $\mathrm{D}=$ Pilo day7; E=Pilo+Lira 12h; F=Pilo+Lira day1; G=Pilo+Lira day3; H=Pilo+Lira day7; I=untreated.

Fig. 4: Number of Bcl-2 positive cells in area CA1 of the hippocampus. A two-way ANOVA found an overall difference between drug treatment and time, and a Tukey repeated measures post-hoc test found differences between time points and drug treatments. ${ }^{* *}=p<0.001 ;{ }^{* *}=p<0.01 ;{ }^{*}=p<0.05$. Shown are representative micrographs. Scale bar $=40 \mu \mathrm{m} . \mathrm{A}=$ Pilo $12 \mathrm{~h} ; \mathrm{B}=$ Pilo day $1 ; \mathrm{C}=$ Pilo day 3 ; $\mathrm{D}=$ Pilo day7; E=Pilo+Lira 12h; F=Pilo+Lira day1; G=Pilo+Lira day3; H=Pilo+Lira day7; I=untreated.

Fig. 5: Number of BAX positive cells in area CA1 of the hippocampus. A two-way ANOVA found an overall difference between drug treatment and time, and a Tukey repeated measures post-hoc test found differences between time points and drug treatments. ${ }^{* * *}=p<0.001 ;{ }^{* *}=p<0.01 ;{ }^{*}=p<0.05$. Shown are representative micrographs. Scale bar $=40 \mu \mathrm{m}$. $\mathrm{A}=$ Pilo $12 \mathrm{~h} ; \mathrm{B}=$ Pilo day1; C=Pilo day 3; $\mathrm{D}=$ Pilo day7; E=Pilo+Lira 12h; F=Pilo+Lira day1; G=Pilo+Lira day3; H=Pilo+Lira day7; I=untreated.

Fig. 6: Levels of key biomarkers in the brain. A one-way ANOVA found an overall difference between groups, and a Tukey repeated measures post-hoc test found differences between time points and drug treatments. ${ }^{* * *}=p<0.001 ;{ }^{* *}=p<0.01 ;{ }^{*}=p<0.05$. Shown are sample blots. Each group $n=3$. 